\title{
Ein angebliches Porträt des schwedischen Arztes Nils Rosén von Rosenstein
}

\author{
Von Heinz Goerke, Örebro (Schweden)
}

Wenige schwedische Ärzte haben für die Medizin ihres Landes so hervorragend gewirkt wie NiLs Rosén, geadelt 1762 unter dem Namen RosÉN von Rosenstein (1706-1773), dessen Buch über die Kinderkrankheiten ${ }^{1}$ seinen Namen weit über die Grenzen seiner Heimat hinaus bekannt gemacht hat. Neben CARL von Linné, dessen Bedeutung als praktizierender Arzt vielfach hinter seinen unvergleichlichen Leistungen als Botaniker übersehen wird, war er es, der in engem Kontakt mit namhaften europäischen Gelehrten stand und deren geachteter Korrespondent war. Persönliche Verbindungen hatte er auf seiner großer Studienreise angeknüpft, die ihn 1729/30 zuerst nach $\mathrm{Halle}^{2}$, wo er fast ein Jahr lang bei FrIEDRICH Hoffmanv hörte, und dann über Kassel, Frankfurt am Main nach Straßburg, in die Schweiz, wo er mit Haller zusammentraf, nach Südfrankreich, Paris und schließlich nach Holland führte. In Harderwijk hat er 1730 mit einer Dissertation über das Schreiben von Krankengeschichten ${ }^{3}$ die medizinische Doktorwürde errungen. Durch eifrigen lebenslangen Briefwechsel sind die Beziehungen zu den gelehrten Ärzten des europäischen Kontinents, vor allem zu BoerhaAve, Haller, van Swieten, aber auch zahlreichen anderen, aufrechterhalten worden und haben zu einem fruchtbringenden Austausch von Gedanken, akademischen Schriften und Buchpublikationen in beiden Richtungen geführt.

Es kann hier nicht mehr über das Leben und wissenschaftliche Werk Roséns gesagt werden, dies soll in anderem Zusammenhang geschehen, nur darauf darf hingewiesen werden, daß sein vorerwähntes Werk über die Kinderkrankheiten in mehrere europäische Sprachen übersetzt worden

1 NiLs Rosén von Rosenstein, Underrättelser om Barn-Sjukdomar och deras Bote-Medel, 2. Auflage, Stockholm 1764. Die erste schwedische Auflage erschien 1753 ff. in den schwedischen Kalendern in Einzelaufsätzen, die 3. Auflage 1771.

${ }^{2}$ Vgl. E. Bergner und H. Goerke, Der schwedische Arzt Nils Rosén über die Medizin an der Universität Halle im Jahre 1729 in Sudhoffs Arch. Gesch. Med. Naturw. 37 (1953) 219-23.

${ }^{3}$ Diss. De historiis morborum conscribendis. Harderwijk 1730. 
ist ${ }^{4}$ und man ihn mit Recht unter die Begründer der Pädiatrie als Sonderfach rechnet. Daß dieses Buch in seiner ersten Auflage in verschiedenen Kalendern erschien, weist auf ein weiteres wichtiges Kennzeichen dieses Gelehrten hin: seinen Wunsch, Verständnis für die Arbeit des Arztes und Kenntnis vom Wesen der Krankheiten als wichtiges Mittel zu ihrer Bekämpfung unter das Volk zu bringen. Diese sozialhygienischen Bestrebungen, die so überaus modern anmuten könnten, waren veranlaßt durch das verhängnisvolle Umsichgreifen des Einflusses unkundiger Quacksalber und Heilkundiger aller Art, die in Schweden sowie im übrigen Europa ihr Unwesen trieben. Welche Bedeutung die Popularisierung der Heilkunde durch Schriften gelehrter Ärzte für die Bekämpfung des Laienbehandlers im 18. Jahrhundert hatte, wäre wohl einer besonderen Untersuchung wert. Man braucht nur an das bekannte Werk Avis au peuple sur la santé des Simon-André Tissot (1728-1797) zu erinnern, dessen erste Auflage 1761 in Lausanne erschien und das man als das Hausarztbuch des Jahrhunderts ansprechen muß ${ }^{5}$. Neben dem Buche Roséns, das ja nur Kinderkrankheiten behandelt und dessen spätere Auflagen den populärmedizinischen Charakter in den Hintergrund treten lassen, sind auch in Schweden einige gute Beispiele für diese Art von Veröffentlichungen nachweisbar ${ }^{6}$.

${ }^{4}$ Deutsche Übersetzung, besorgt von JoH. And. Murray (1740-91), Schwede, von 1764. bis 1791 Professor der Medizin und Botanik in Göttingen, unter dem Titel Anweisung zur Kenntniß und Cur der Kinderkrankheiten, 1. Auflage 1766 (sechs weitere Auflagen, die letzte 1798, nach dem Tode Murrays, von Loder und Buchнolz besorgt). Diese Übersetzung zeichnet sich durch zahlreiche wertvolle Annotationen des Übersetzers aus. Daneben ist noch eine zweite deutsche Übertragung (Wien 1787) bekannt. Übersetzungen in andere europäische Sprachen: eine holländische Auflage (1768), zwei französische $(1778,1792)$, zwei italienische $(1780,1783)$, eine englische (1776) und eine dänische Auflage (1769). Es soll auch noch eine tschechische Übersetzung existieren, von der uns aber bisher kein Exemplar zu Gesicht gekommen ist.

${ }^{5}$ Das Werk erlebte in sechs Jahren zehn Auflagen, ist später in unzähligen Neuauflagen und Übersetzungen in zahlreichen europäischen Sprachen erschienen.

6 Z.B. Јoh. HaArtman (1725-87), Studium in Åbo und Uppsala, Dr. med. in Uppsala 1754, seit 1764 Professor der Medizin in Åbo. Er schrieb Underrättelse om de mäst gångbare sjukdomars igenkännande och botande genom lätta och enfaldiga husmedel, Stockholm 1759, 2. Auflage Åbo 1765. (Mitteilung über das Erkennen der gangbarsten Krankheiten und der Heilung durch leichte und einfältige Hausmittel.)

Joh. And. Darelius, 1770 geadelt AF DARELli (1718-80), Studium in Uppsala, assistierte Rosén in der Praxis, 1749 Dr. med. in Uppsala, 1750 Mitglied des Collegium medicum. Er schrieb Socken-Apothek och någre huscurer, Stockholm 1760, 2. Auflage 1771. Bekannt sind dänische und deutsche Übersetzungen (Gemeinde-Apotheke und einige Hauskuren). 
In die gleiche Richtung tendierte auch die große Zahl der kurzgefaßten therapeutischen Taschenbücher, die als «Haus- und Reise-Apotheken», «Land-Apotheken» oder unter ähnlichen Titeln herausgegeben wurden. Auch hier wurde der des Lesens kundige Laie angesprochen und ihm für Notfälle und Erste Hilfe Rat erteilt. Auch aus der Feder Roséns kennen wir ein solches Büchlein, das auch in einer dänischen und zwei deutschen Übersetzungen bekannt geworden ist ${ }^{7}$. Damit ist aber auch die Zahl seiner populärmedizinischen Veröffentlichungen in Buchform erschöpft.

Um so mehr überrascht waren wir, als uns kürzlich ein Exemplar eines Werkes zu Gesicht kam, ein Hausarztbuch, das in französischer Sprache dem schwedischen Autor von Rosén zugeschrieben wurde ${ }^{8}$. Der volle Titel lautet: Le médecin de la montagne ou le guide des praticiens de campagne et des personnes charitables qui s'employent à secourir les malades: nouvelle médecine et chirurgie des pauvres, Traduit du Suédois, de Roseen, par Mr. C. MÉdecin; Précédé d'une introduction par P.V., Médecin de la Faculté de Montpellier. A Lyon, chez les frères Perisse, Imprimeur-Libraires, grande rue Merciere, No. 15. An. 12. 1803. Das Werk stellt eines der üblichen Hausarztbücher der Zeit dar, ist in Oktav gedruckt und behandelt auf 345 Seiten folgende Themen: die Fieber, die Krankheiten des Kopfes, der Brust, des Bauches, der Glieder und chirurgische Vorfälle (143 Seiten). Angefügt ist ein elfseitiges Verzeichnis von einfachen Arzneimitteln. Ganz sicher würde sich in Schweden, dessen Bibliotheksbestände sich durch erstaunliche Vollkommenheit auszeichnen, ein Exemplar der schwedischen Auflage dieses Werkes finden, falls Rosén es geschrieben hätte. Aber weder von ihm noch von einem anderen schwedischen Autor ist ein Buch solchen Inhaltes geschrieben worden, daß seine französische Übersetzung dem vorliegenden Exemplar entsprechen könnte. Hat man sich etwas mehr darin vertieft, so scheint einem nach Form und Inhalt der dort vermittelten medizinischen Gedanken die Verfasserschaft eines Skandinaviers nahezu ausge-

${ }^{7}$ Hus- och Reseapoteque på Hennes Kongl. Majts. nådigste befallning upsatt (Haus- und Reiseapotheke, auifgestellt auf Geheiß Ihrer Majestät der Königin) Stockholm 1765, 2. Auflage 1772. Eine dänische Auflage 1768, zwei deutsche Übersetzungen nach verschiedenen schwedischen Auflagen, davon eine von JoH. AND. Murray 1781 nach der 2. schwedischen Auflage besorgt.

${ }^{8}$ Das Buch ist im Besitz des bekannten Bibliophilen und um die schwedische Medizingeschichte verdienten früheren Chefarztes der Frauenklinik am Zentralkrankenhaus in Örebro (Schweden), Dr. Erik Bergner, dem ich für die freundliche Überlassung desselben zum Zwecke der Bearbeitung verbindlichst danke. 
schlossen zu sein und ein französischer Autor viel eher in Betracht zu kommen. Das zweifellos seltene Buch, Umfrage bei einer Reihe von großen Bibliotheken des Kontinents wurde mit «nicht vorhanden » beantwortet, stellt offenbar das Werk eines anonymen Verfassers dar, dessen Verleger den Namen des schwedischen Gelehrten als besonders zugkräftig ansah. Ein Beweis, wie sehr der Name dieses Uppsalienser Professors der Medizin, noch dreißig Jahre nach seinem Tode, mit medizinischer Gelehrsamkeit und medizinischer Volksaufklärung verbunden war.

Von ganz besonderem Interesse aber ist ein dem Buch vorgehefteter Stich (Abb. 1), der als Porträt des angeblichen Verfassers bezeichnet wird und den man ohne weiteres als Fälschung erkennt. Jeder, der eines der zahlreichen Bilder Nils Roséns (Abb.2) kennt, sieht, daß man es hier nicht mit dem bekannten schwedischen Arzt zu tun hat. Der hier als «Roseen Medecin Suedois» Bezeichnete ist niemand anders als der Schweizer «Bergdoktor» - interessant der damit übereinstimmende Buchtitel Médecin de la montagne - Michael Schüppach (1707-1781) aus Langnau. Das bekannte Porträt Schüppachs (Abb. 3) ${ }^{9}$, der im Original als «Michael Schuppach, Médecin Praticien très renommé à Langnau dans le Canton de Berne ${ }^{10}$ bezeichnet wird, ist seitenverkehrt und mit veränderter Staffage verwandt worden. Bei Erscheinen des Buches war auch Schüppach bereits zweiundzwanzig Jahre tot und sein Bild in Frankreich sicher nicht so bekannt, als daß die Bezeichnung desselben als eine Darstellung Roséns verfänglich gewesen wäre.

Zweck dieses kurzen Aufsatzes sollte sein, auf ein Porträtfalsifikat hinzuweisen, das ebenso wie die Verwendung des Namens Rosén als Autor des Médecin de la montagne auf den bedeutenden Ruf hinweist, den dieser schwedische Arzt auch außerhalb seines Landes genossen hat.

${ }^{9}$ Die Vorlage des Rosén-Bildes, ein Stich von G. Benoist, verdanke ich der Porträtsammlung der Königlichen Bibliothek in Stockholm, die des Porträts von Schüppach der Freundlichkeit der Burgerbibliothek in Bern.

${ }^{10}$ Originalporträt nicht bekannt, gestochen bei Basant und Poignant, Paris (vgl. Sammlung Bernischer Biographien, herausgegeben vom Historischen Verein des Kantons Bern, Bd. II, Bern 1896, S. 204). 


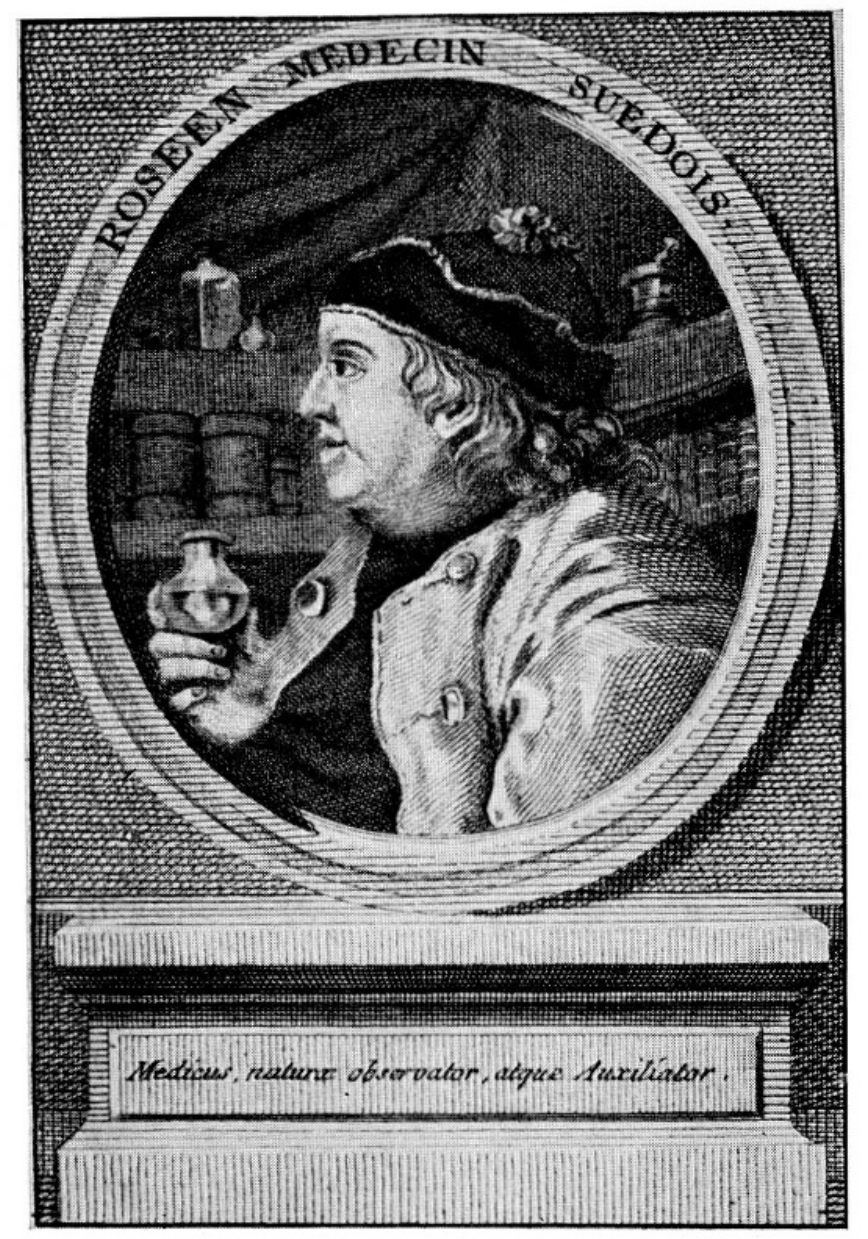

Abb. 1

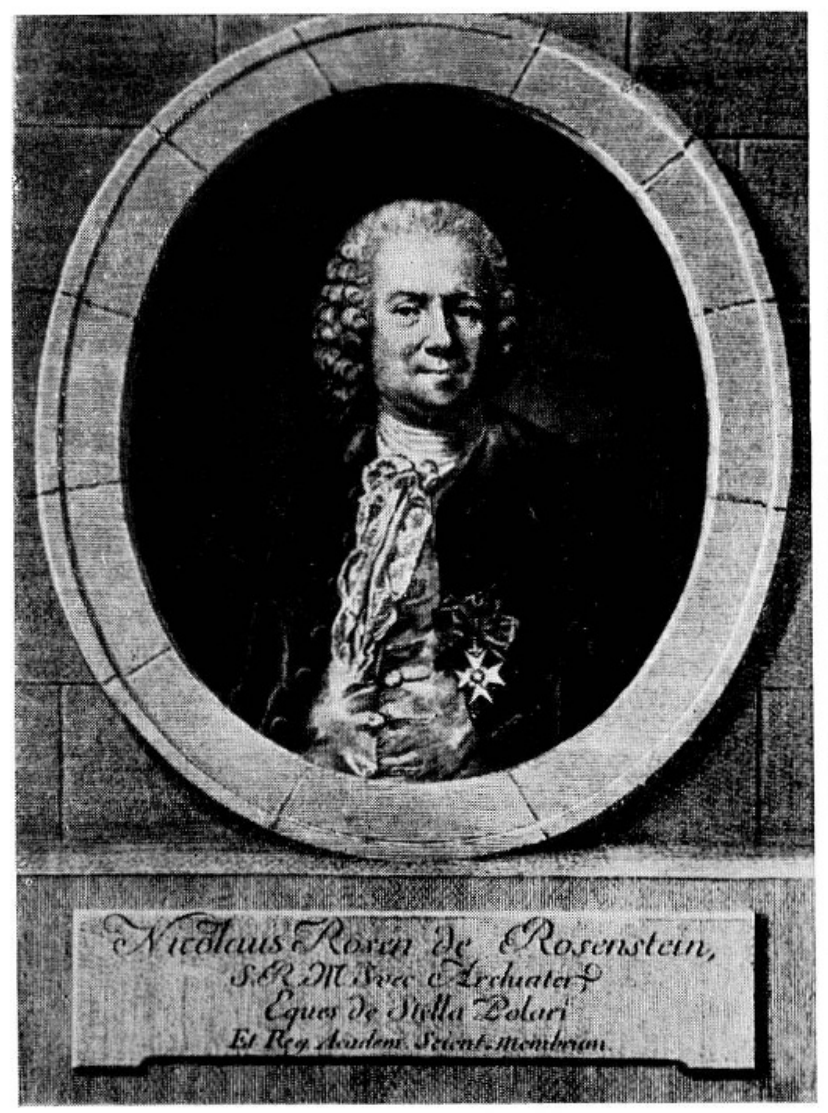

Abb. 2

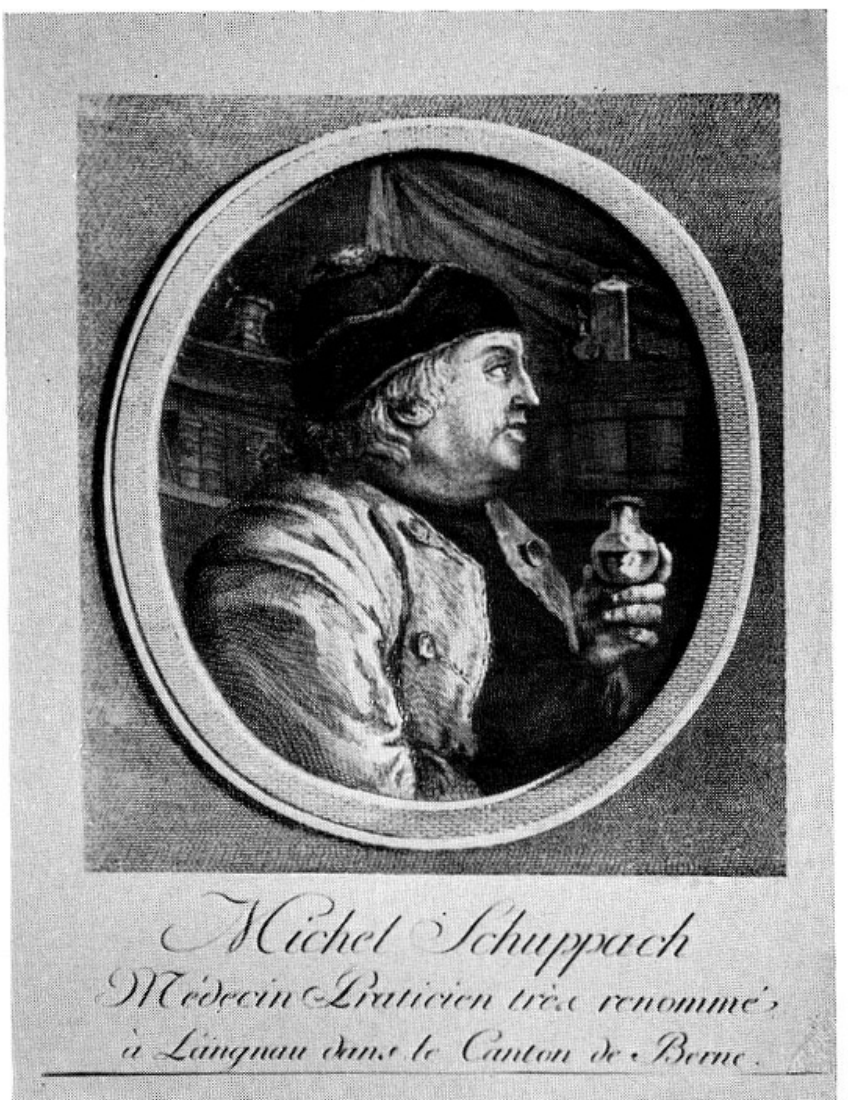

Abb. 3 